\title{
Contraception and unexplained abdominal pain
}

\author{
Martin Vessey, MD, FRS, Emeritus Professor of Public Health; Rosemary Painter, DPhil, Computer Scientist, Institute of Health \\ Sciences, Oxford, UK
}

Correspondence: Martin Vessey, Unit of Health Care Epidemiology, Oxford University Department of Public Health, Institute of Health Sciences, Headington, Oxford OX3 7LF, UK. Email: martin.vessey@dphpc.ox.ac.uk

(Accepted $12^{\text {th }}$ September 2001)

The Journal of Family Planning and Reproductive Health Care 2002: 28(1): 32-33

\begin{abstract}
Objective. To test the hypothesis that hospital referral for unexplained abdominal pain might occur less frequently in oral contraceptive (OC) users and more frequently in intrauterine device (IUD) users than in other women.

Design. Prospective cohort study of 17032 women using different methods of contraception [the Oxford-Family Planning Association (FPA) contraceptive study].

Outcome measure. Referral to hospital for unexplained abdominal pain (including pelvic pain) coded to ICD rubric 785.5 in the 8th Revision of the ICD.

Results. Referral was decreased by 13\% (95\% CI 0\%-25\%) in current or recent users of OCs in comparison with nonusers, a difference that just reached statistical significance. No significant difference was found in the corresponding analysis for IUD users. Past users of OCs had much the same risk of referral as non-users, but the risk of referral in past users of an IUD was increased at all intervals examined, varying from $17 \%(95 \%$ CI -14\%-55\%) $13-24$ months after discontinuation to 56\% (95\% CI 15\%-107\%) 73-96 months after discontinuation. Referral was $29 \%$ (95\% CI 11\%-50\%) higher in smokers of 15 or more cigarettes a day than in non-smokers.

Conclusion. Referral to hospital for unexplained abdominal pain may be slightly reduced in current or recent OC users and moderately increased in past IUD users. The types of IUD used most commonly in the Oxford-FPA study are, however, no longer in current use today. Hospital referral for unexplained abdominal pain seems to be moderately increased in heavy cigarette smokers.
\end{abstract}

\section{Key message points}

- Hospital referral for unexplained abdominal pain in the Oxford-FPA study was slightly decreased in current or recent users of oral contraceptives (OCs) in comparison with non-users. No corresponding difference was found in a similar analysis for intrauterine device (IUD) users.

- Past users of OCs had much the same risk of hospital referral for unexplained abdominal pain as non-users. The risk of referral in past users of an IUD, however, was increased at all intervals examined. It should be remembered that the types of IUD most commonly used in the Oxford-FPA study are no longer in use today.

- The risk of referral was moderately increased in heavy (15 or more cigarettes a day) smokers.

\section{Introduction}

Unexplained abdominal pain (including pelvic pain) is a common diagnosis among women in the Oxford-Family Planning Association (Oxford-FPA) contraceptive study. Oral contraceptive (OC) use decreases the risk of a number of causes of abdominal pain including dysmenorrhoea, ${ }^{1}$ functional ovarian cysts ${ }^{2}$ and pelvic inflammatory disease. ${ }^{3}$ The risk of endometriosis ${ }^{4}$ and uterine fibroids ${ }^{5}$ may also be reduced in pill users. Use of an intrauterine device (IUD), however, especially one of the older types widely represented in the Oxford-FPA study, increases the risk of dysmenorrhoe ${ }^{6}$ and pelvic inflammatory disease. ${ }^{3}$ In view of these observations and the difficulty often experienced in diagnosing the conditions listed, we hypothesised that unexplained abdominal pain would occur less frequently in OC users and more frequently in IUD users than in other women. We present our findings here.

\section{Methods}

The Oxford-FPA study methods have been described elsewhere. ${ }^{7}$ Briefly, 17032 white married women, aged 25-39 years, using OCs, a diaphragm or an IUD were recruited at 17 family planning clinics in the UK between 1968 and 1974. Information about socio-medical variables was collected at admission. Information collected during follow-up, which took place at annual intervals until 1994, included changes in contraceptive practices and reasons for hospital referral. Discharge summaries were obtained for hospital admissions. For outpatient consultations, diagnoses reported by the women themselves were generally accepted. Coding of the information collected was done at Oxford, the diagnostic coding being done by one of us (MV) throughout. Losses to follow-up for reasons that might cause bias were kept to a low level (about four women per 1000 per year). When women reached the age of 45 years, they were divided into three groups - those never using OCs, those using them for 8 or more years, and the remainder. Detailed follow-up as described above continued only for women in the first two groups.

An episode of unexplained abdominal pain (including pelvic pain) was identified as any referral carrying the diagnostic code 785.5 (abdominal pain not otherwise specified) within the 8th Revision of the ICD. Only the first episode for each woman was considered in the analysis.

Analyses were based on woman years of observation in the groups of interest with calculation of indirectly standardised rates. ${ }^{7}$ The computation of $95 \%$ confidence intervals (CI) around estimates of relative risk was based on methods described by Breslow and Day. ${ }^{8}$

\section{Results}

In total, 1600 women were referred to hospital with a first episode of unexplained abdominal pain (5.5 per 1000 woman years; 95\% CI 5.2-5.8). The first step in the analysis was to identify possible confounding factors. The 
variables examined were age, parity, social class, cigarette smoking, height and weight. Referral risk did not vary much with any of these variables although it was slightly higher in women aged 35-44 years than in older or younger women. In addition, in comparison with non-smokers, the relative risk was elevated among those smoking 15 or more cigarettes per day $(1.29 ; 95 \%$ CI $1.11-1.50)$

In examining the data by contraceptive method, risks were adjusted for age and smoking. No significant trends in referral risk were found with total duration of use of OCs or IUDs. Likewise, there was little variation in risk in relation to the interval since OCs were last used (Table 1). Nonetheless, the risk for those currently or recently using OCs was reduced by $13 \%$ in comparison with non-users; this difference just reached the $5 \%$ level of statistical significance. Past users of an IUD had considerably higher risks of referral than current or recent users or non-users in all groups shown in Table 1; figures for 73-96 months and 97 or more months reached statistical significance.

Table 1 Hospital referrals for unexplained abdominal pain in relation to interval since last use of an oral contraceptive or an intrauterine device ${ }^{a}$

\begin{tabular}{lll}
\hline Interval since last use & $\begin{array}{l}\text { Oral } \\
\text { contraceptive (OC) }\end{array}$ & $\begin{array}{l}\text { Intrauterine } \\
\text { device (IUD) }\end{array}$ \\
\hline Non-user & 1.00 & 1.00 \\
Current or in last 12 months & $0.87(0.75-1.00)$ & $0.95(0.82-1.11)$ \\
$13-24$ months & $0.96(0.71-1.29)$ & $1.37(0.93-1.94)$ \\
25-48 months & $1.08(0.88-1.34)$ & $1.17(0.86-1.55)$ \\
$49-72$ months & $1.22(1.00-1.51)$ & $1.31(0.96-1.74)$ \\
$73-96$ months & $1.12(0.89-1.42)$ & $1.56(1.15-2.07)$ \\
$97+$ months & $1.01(0.85-1.20)$ & $1.30(1.04-1.62)$ \\
\hline
\end{tabular}

aData are relative risks adjusted for age and cigarette smoking with $95 \%$ confidence intervals.

Age groups (years) used in adjustment: 25-29, 30-34, 35-39, 40-44, 45-49, 50+.

Smoking groups used in adjustment: never, ex-smoker, 1-14 cigarettes/day, $15+$ cigarettes/day.

In addition to our main analysis, we also studied the 1200 women remaining after we had identified and excluded those who had had a hospital referral for a relevant specific condition during the year before or the year after the episode of unexplained abdominal pain. Relevant infections, neoplasms, gastrointestinal diseases, urinary tract diseases and diseases of the genital tract were taken into account (a list of the conditions considered is available from the authors on request). The findings were much as in Table 1, although slightly less extreme with regard to IUD use.

\section{Discussion}

Women with unexplained abdominal pain are not a homogeneous diagnostic group; often the label merely implies that the clinician was satisfied that no sinister condition was the underlying cause. In addition to our main analysis involving 1600 women we also examined a subgroup of 1200 women with no known relevant specific diagnosis during the year before or the year after their episode of pain. Findings were much the same in both groups.

The association between heavy cigarette smoking and unexplained abdominal pain was unexpected. We have previously reported a relationship between smoking and unexplained chest pain. ${ }^{9}$ We judged this finding to be due to occult heart and lung disease in smokers. Our present finding is less easy to explain, although disease affecting the thoracic organs may sometimes manifest itself as abdominal pain. In addition, general practitioners (GPs) may be more likely to refer to hospital women with either chest or abdominal pain who smoke, fearing a more sinister condition in smokers than in non-smokers.

Little relationship was found between OC use and unexplained pain, although current or recent users were at a slightly reduced relative risk of referral in comparison with non-users $(0.87 ; 95 \%$ CI $0.75-1.00)$. Nonetheless a reduction of this magnitude, if attributable to OC use, would be of some clinical significance, representing an annual reduction in referrals of about 0.7 women per 1000 .

Our main finding is an excess of referrals in past users of an IUD, but not in current or recent users. This observation is not easy to interpret, although findings in the Oxford-FPA study on hospital referral for pelvic inflammatory disease ${ }^{3}$ may offer a partial explanation at least. Current users of a non-copper-bearing IUD had a relative risk of 3.3 (95\% CI 2.3-5.0) and of a copper-bearing IUD of 1.8 (95\% CI $0.8-4.0)$ in comparison with the control group. The relative risk in past users of an IUD was 1.3 (95\% CI 0.7-2.3). It seems possible that pelvic inflammatory disease is recognised more readily in current or recent users of an IUD (and coded as such) than it is in past users of an IUD and this might have contributed to the present findings.

Neither the Royal College of General Practitioners Oral Contraception Study ${ }^{10}$ nor the Walnut Creek Contraceptive Study ${ }^{11}$ has reported data concerning unexplained abdominal pain and we are unaware of other publications relevant to the present analysis. We conclude that a reduction of about $13 \%$ in hospital referrals for unexplained abdominal pain may possibly be another benefit of current or recent OC use, but that there seems to be an increase in the risk of such referrals of about $35 \%$ in past users of an IUD that might be related in part to unrecognised pelvic inflammatory disease. It is important to remember, however, that the types of IUD most commonly used in the Oxford-FPA study are no longer in use today.

\section{Acknowledgements}

We would like to thank Mrs J Winfield, our research assistants, and the staff at the participating clinics for their contributions to this project.

\section{Statements on funding and competing interests}

Funding. The Oxford-FPA study is funded by a grant from the Medical Research Council.

Competing interests. None declared.

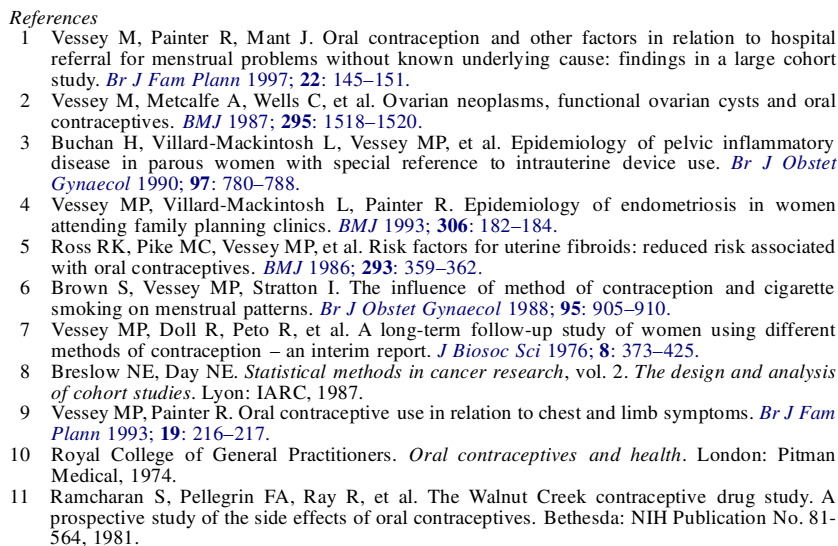
cout known underlying cause: findings in a large colo study. Br J Fam Plann 1997; 22: 145-151.

Study. Br M, Metcalfe A, Wells C, et al. Ovarian neoplasms, functional ovarian cysts and ora
Vessey M, contraceptives. BMJ 1987; 295: 1518-1520.

Buchan $\mathrm{H}$, Villard-Mackintosh L, Vessey MP, et al. Epidemiology of pelvic inflammatory disease in parous women with special reference to intrauterine device use. $\mathrm{Br} J$ Obste Gynaecol 1990; 97: 780-788.

Vessey MP, Villard-Mackintosh L, Painter R. Epidemiology of endometriosis in women attending family planning clinics. BMJ 1993 ; 306: $182-184$

tors for uterine fibroids: reduced risk associated Ross RK, Pike MC, Vessey MP, et al. Risk $359-362$.
with oral contraceptives. $B M J$ 1986; 293: 359-

Brown S, Vessey MP. Stratton I. The influence of method of contraception and cigarette smoking on menstrual patterns. Br J Obstet Gynaecol 1988; 95: 905-910.

Vessey MP, Doll R, Peto $R$, et al A long-term follow-up study of women using different methods of contraception - an interim report. I Biosoc Sci 1976; 8: $373-425$.

Breslow NE, Day NE. Statistical methods in cancer research, vol. 2. The design and analysis of cohort studies. Lyon: IARC, 1987.

Vessey MP, Painter R. Oral contraceptive use in relation to chest and limb symptoms. Br J Fan Plann 1993; 19: 216-217.

10 Royal College of General Practitioners. Oral contraceptives and health. London: Pitman

Ramcharan S, Pellegrin FA, Ray R, et al. The Walnut Creek contraceptive drug study. A the side effects of oral contraceptives. Bethesda: NIH Publication No. 81564,1981 